\title{
Outcomes of young people who reach the transition boundary of child and adolescent mental health services: a systematic review
}

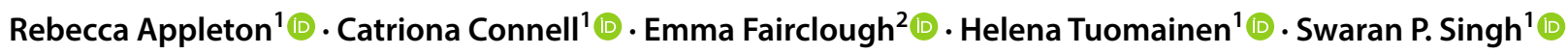

Received: 16 October 2018 / Accepted: 2 March 2019 / Published online: 8 March 2019

(c) The Author(s) 2019

\begin{abstract}
When young people reach the upper age limit of child and adolescent mental health services (CAMHS), care should be transferred to an adult mental health service (AMHS) if they require ongoing support. However, many young people experience a significant disruption of their care during this transition, whilst others may fail to transition at all. Currently, there is no systematic appraisal of the international evidence regarding the outcomes of young people after transition. A systematic review was conducted which aimed to synthesise and review the existing research regarding outcomes after transition. We searched six databases from their inception until December 2017 for research relating to either the mental health or service use outcomes of young people after reaching their CAMHS age boundary. Results were synthesised narratively. The initial searches identified 18,287 papers, of which 213 were screened on full text. 13 papers were included in the review, representing 10 cohorts of young people who crossed the transition age boundary. No studies contained extractable data on mental health outcomes following transition, and therefore, this review focused only on service use outcomes. Results showed a quarter of young people transitioned to AMHS, with the other young people experiencing varied outcomes after leaving CAMHS and multiple transitions during this time. This review provides evidence for the varying service use outcomes of young people after reaching the upper age limit of CAMHS. However, longitudinal research into long-term outcomes is lacking, in addition to research regarding the mental health and functioning outcomes of young people following transition. Protocol registration The protocol for this systematic review has been registered with PROSPERO, ID number CRD42018085916.
\end{abstract}

Keywords Transition $\cdot$ CAMHS $\cdot$ AMHS $\cdot$ Mental health service $\cdot$ Systematic review

\section{Introduction}

In high-income countries, mental health services are divided into separate specialties for children and adolescents, and adults. When young people reach the upper age limit of their child and adolescent mental health service (CAMHS), at around the age of 16-18 [1, 2], decisions need to be made regarding their future care. If a young person approaching this age boundary is judged to have an ongoing clinical need which requires specialist future treatment, care should be transferred to an adult mental health service (AMHS). This

Rebecca Appleton

b.appleton@warwick.ac.uk

1 Warwick Medical School, University of Warwick, Coventry CV4 7AL, UK

2 Institute of Psychological Sciences, University of Leeds, Leeds LS2 9JT, UK transfer of care should occur through part of the therapeutic process known as transition [3]. Four features of optimal transition have been identified: it should be planned well in advance and feature a joint meeting between both clinical teams and the young person; there should be a period of parallel care; all the young person's information should be transferred to the new service and there should be continuity of care after the young person has left CAMHS [4]. However, the previous research has shown that these four features rarely occur, with one study estimating them to be present in only $4 \%$ of transitions [4].

Whilst some find the transition to AMHS difficult, some young people fail to transition at all, despite having an ongoing clinical need [5]. Some young people, although unwell, may not meet the eligibility thresholds for care at AMHS [6], which are often higher than those to access care at CAMHS. These differences in threshold are partly due to the differing approaches between the services [7], with CAMHS focusing 
on developmental and family problems, and AMHS specialising in the treatment of more severe chronic mental illnesses $[8,9]$. The contrasting approaches between the services can also make it difficult for young people to adapt to care at AMHS [10], which could lead to poor engagement with their new service [11].

Young people who do not successfully transition to AMHS despite still needing care are said to have fallen through the gap between services. Currently, we do not know what happens to these young people, something which has been called a "serious cause for concern" [4] (p310) and highlighted as a significant gap in our knowledge in a recent report by the National Health Service (NHS) Healthcare Safety Investigation Branch [12] and in National Institute for Health and Care Excellence (NICE) transition guidelines [13].

To the best of our knowledge, no study has attempted to systematically review and robustly collate the evidence regarding the clinical and functioning outcomes (e.g., illness severity and living skills) of young people once they have reached the upper limit of their CAMHS service. The research questions for this review are as follows: (1) what are the service use destinations of young people after they reach the CAMHS age boundary? (2) what are the mental health outcomes of young people after they reach the CAMHS age boundary?

\section{Methods}

This systematic review was conducted and reported in concordance with the PRISMA guidelines. The protocol for this review was registered with PROSPERO, ID number CRD42018085916.

\section{Search strategy}

After the initial scoping searches, six bibliographic databases were searched (Medline, PsycINFO, CINAHL, Embase, and Web of Science) for the relevant literature from their inception until December 2017. Search terms were developed in collaboration with an information specialist, and contained terms relating to transition, young people, and mental health. An example search strategy can be found in Table 1 . The reference lists of relevant systematic reviews which were identified during title and abstract screening were hand searched for additional relevant studies, although none were identified.
Table 1 An Example search strategy from Medline

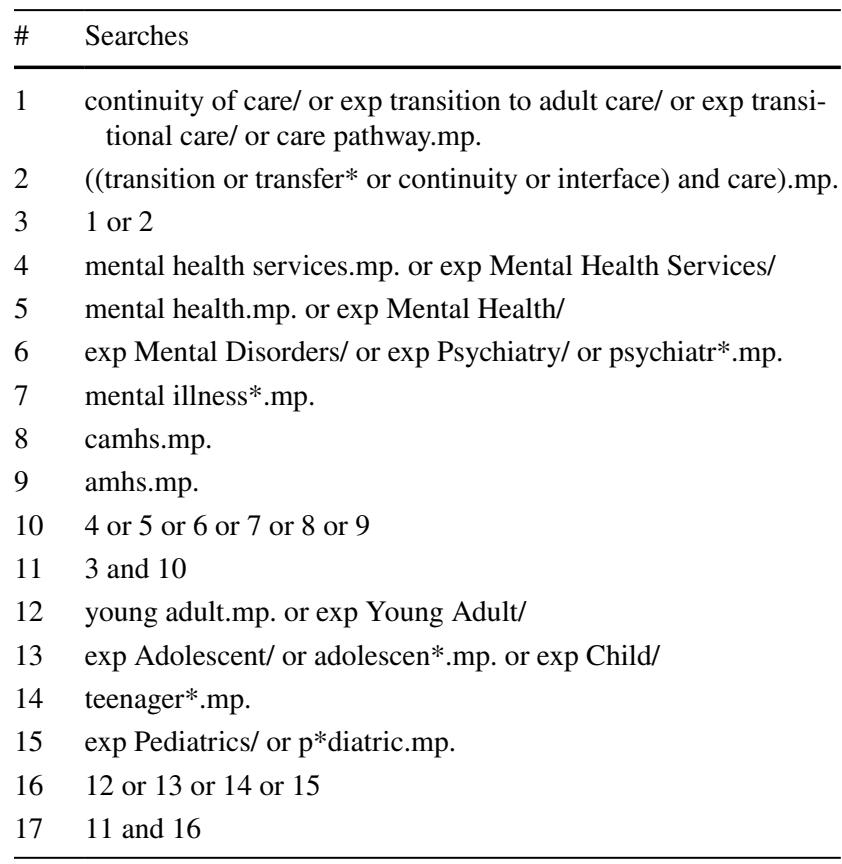

\section{Eligibility criteria}

Studies were eligible to be included if they provided details of the clinical or functional outcomes of a cohort of young people (from mid-late adolescence to early adulthood) who crossed the transition boundary of children's mental health services, or if they provided details of the service pathway taken by a cohort of young people who crossed the transition boundary. Here, we define transition boundary as the upper age limit of a CAMHS. Conference abstracts were eligible to be included if the research had not been published elsewhere. There were no language restrictions in this review.

We did not include research involving the transition of young people with physical illnesses, neurological conditions (e.g., epilepsy), young people with a severe learning disability, or young people who were not transitioning in a mental health service. Case studies, editorials, literature or systematic reviews, opinion pieces, and policy documents were also excluded.

\section{Study selection}

After de-duplication of references, titles and abstracts were screened by one reviewer (RA), and a random $10 \%$ were screened by another member of the research team (EF). Agreement was high between both reviewers $\left(k_{\max }=0.85\right)$. Any references which met the inclusion criteria were then 
screened by full text by two reviewers independently (split between RA, EF, and CC). If the title and abstract did not contain sufficient information to decide on eligibility, then they were included for full-text screening. Any disagreement between reviewers was resolved through discussion.

\section{Quality assessment}

Quality assessment of included studies was conducted independently by two reviewers (RA and CC) using a modified version of the Newcastle-Ottawa Scale [14]. All studies were included regardless of quality due to the lack of research in this area; however, the results of quality assessment were used to inform the narrative synthesis of results.

\section{Data extraction}

A data extraction tool was piloted on a small number of included studies, modified, and then used to extract data from all studies. It included the following headings: year of publication, country of origin, aims, study design, sampling method, methodology, results, and how results were presented. Data extraction was carried out by two reviewers independently (RA and CC).

\section{Data synthesis}

Data were synthesised narratively using steps adapted from Popay et al. [15]. These are: (1) to develop a preliminary synthesis of findings of included studies; (2) to explore relationships in the data; (3) to assess the robustness of the synthesis. A meta-analysis was not conducted due to the heterogeneity of the included studies.

\section{Results}

\section{Study selection}

After duplicates were removed, 18,287 studies remained for screening by title and abstract. 213 studies were included for full-text screening, of which 200 studies were excluded to leave 13 studies for inclusion in this review, representing 10 different cohorts of young people crossing the CAMHS transition boundary. Figure 1 illustrates the paper selection process. Only one study explored mental health outcomes after transition [16]; however, this data could not be extracted as CAMHS leavers were grouped with looked after children. Therefore, only information on service use outcomes following transition will be discussed in this review.

\section{Study characteristics}

The 13 included studies represent research carried out in six different countries, Canada [17], England [2-4, 16, 18, 19], the Republic of Ireland [5, 20], France [21], Australia [22], and Italy $[23,24]$. Two studies were service evaluations [17, 19]: one was a questionnaire study [24], one was a longitudinal study [16], and the remaining nine had a retrospective cohort study design $[2-5,18,20-23]$. Seven of the studies involved all young people in a cohort of CAMHS leavers, whilst four focused only on young people with attentiondeficit/hyperactivity disorder (ADHD) [2, 19, 20, 24]. The sample sizes in the included studies ranged from 20 to 4226 young people. Table 2 shows further details of the included studies.

\section{Risk of bias}

The quality of the included studies varied, with 10 being of good quality and three being of poor quality (see Table 2 for more details). Studies were rated as poor if they did not include a measure of clinical need to transition or a breakdown of transition for different subgroups (e.g., different diagnoses, age groups, severity of illness, etc.) and if detailed baseline information of the cohort was missing.

\section{Synthesis of results}

The synthesis of individual study findings shows a care gap at the end of CAMHS, with only $24 \%$ of young people transitioning to AMHS after reaching their CAMHS age boundary (see Table 3 for details). Three studies $[4,5,16]$ explored the service use destinations of young people who had an ongoing clinical need at the end of CAMHS and found that some did not receive an AMHS referral, despite still being judged to need ongoing care, with figures ranging from 42 to $84 \%$ (the latter figure includes some looked after children in Memarzia et al. [16]). In addition, four studies [19, 20, $22,24]$ showed that 103 young people were discharged from CAMHS, only for them to be referred to AMHS by their GP.

A quarter of young people remained at CAMHS after crossing the transition boundary, whilst another quarter transitioned to AMHS. The other 50\% had varied service use destinations; however, in most studies, the follow-up periods were not long enough to find out what happened to these young people after being discharged from CAMHS. Disengagement was high, with all but four studies [16, 22-24] including disengagement as an outcome after young people left care at CAMHS. The number of young people who were discharged due to disengagement was recorded in all but one study [2], with disengagement ranging from 3 to $40 \%$ of young people. 
Fig. 1 PRISMA flowchart showing screening of identified papers

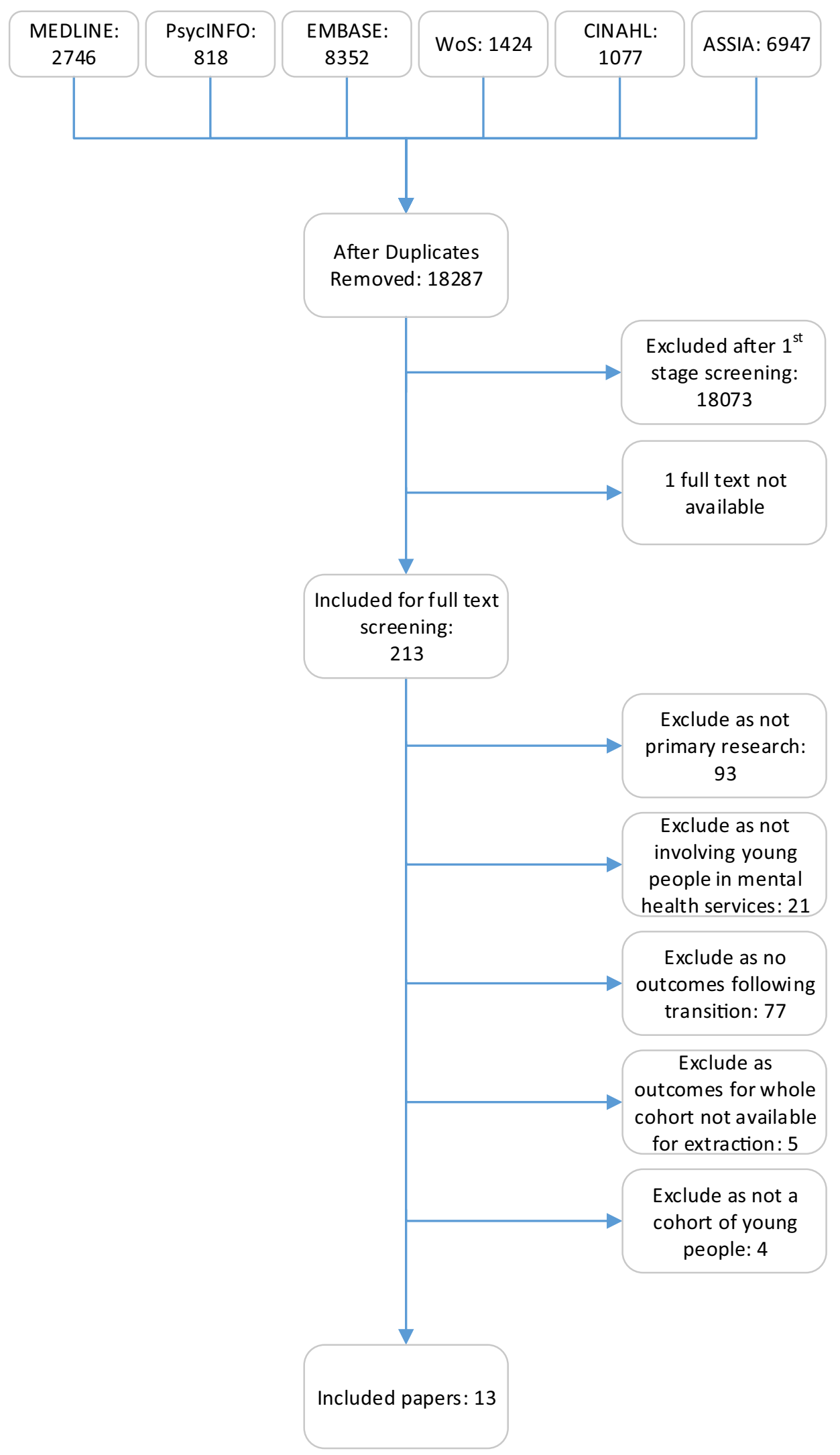




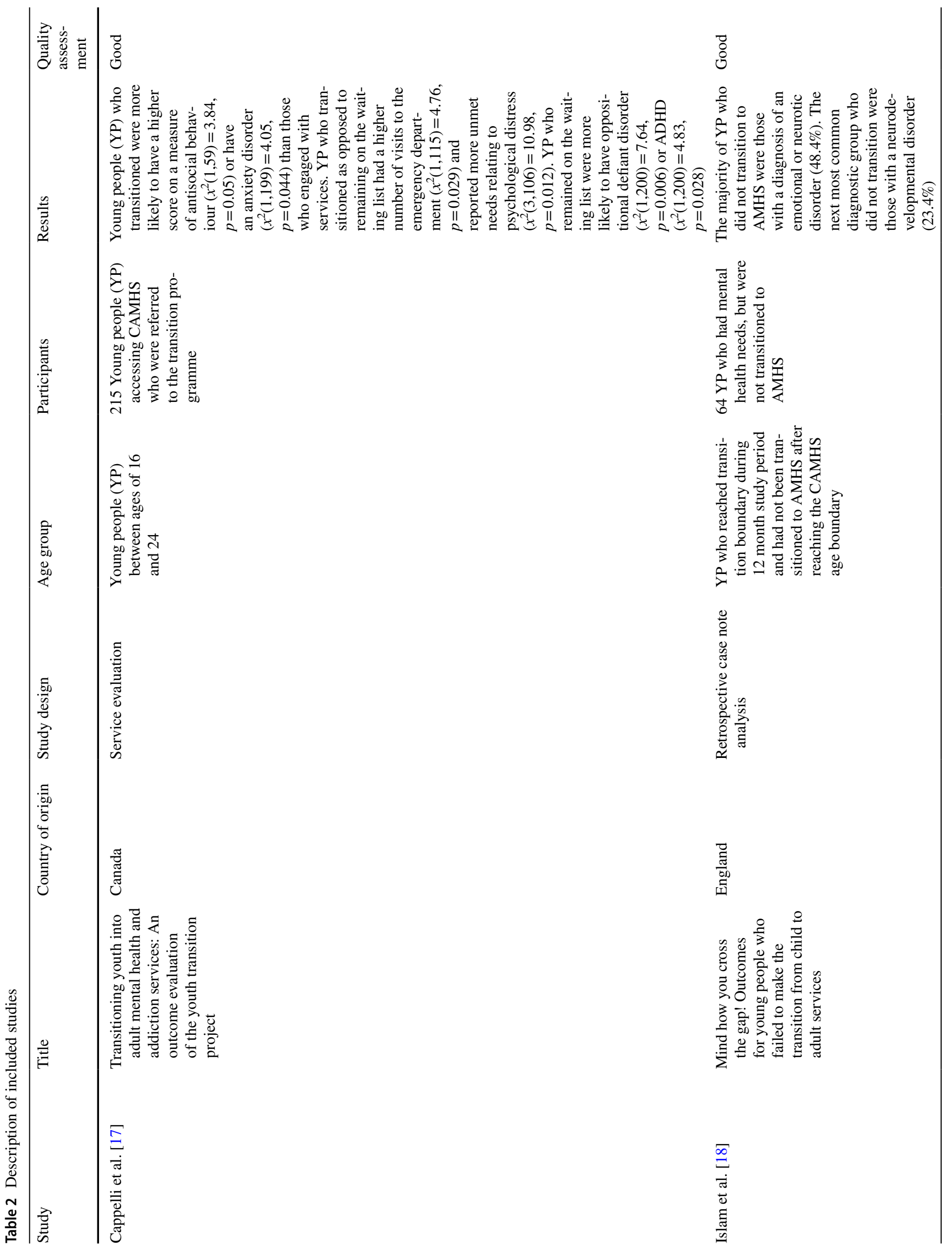




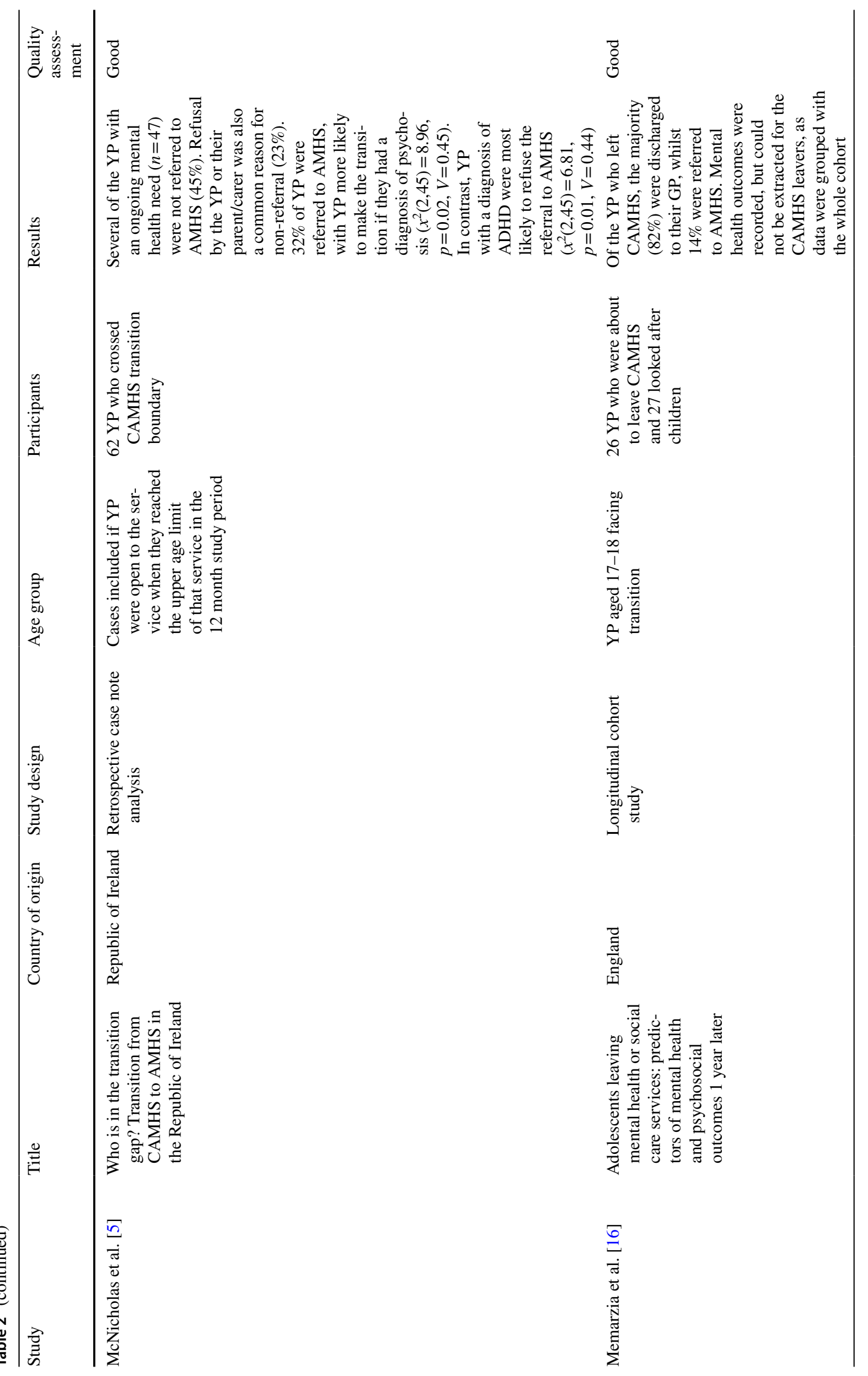




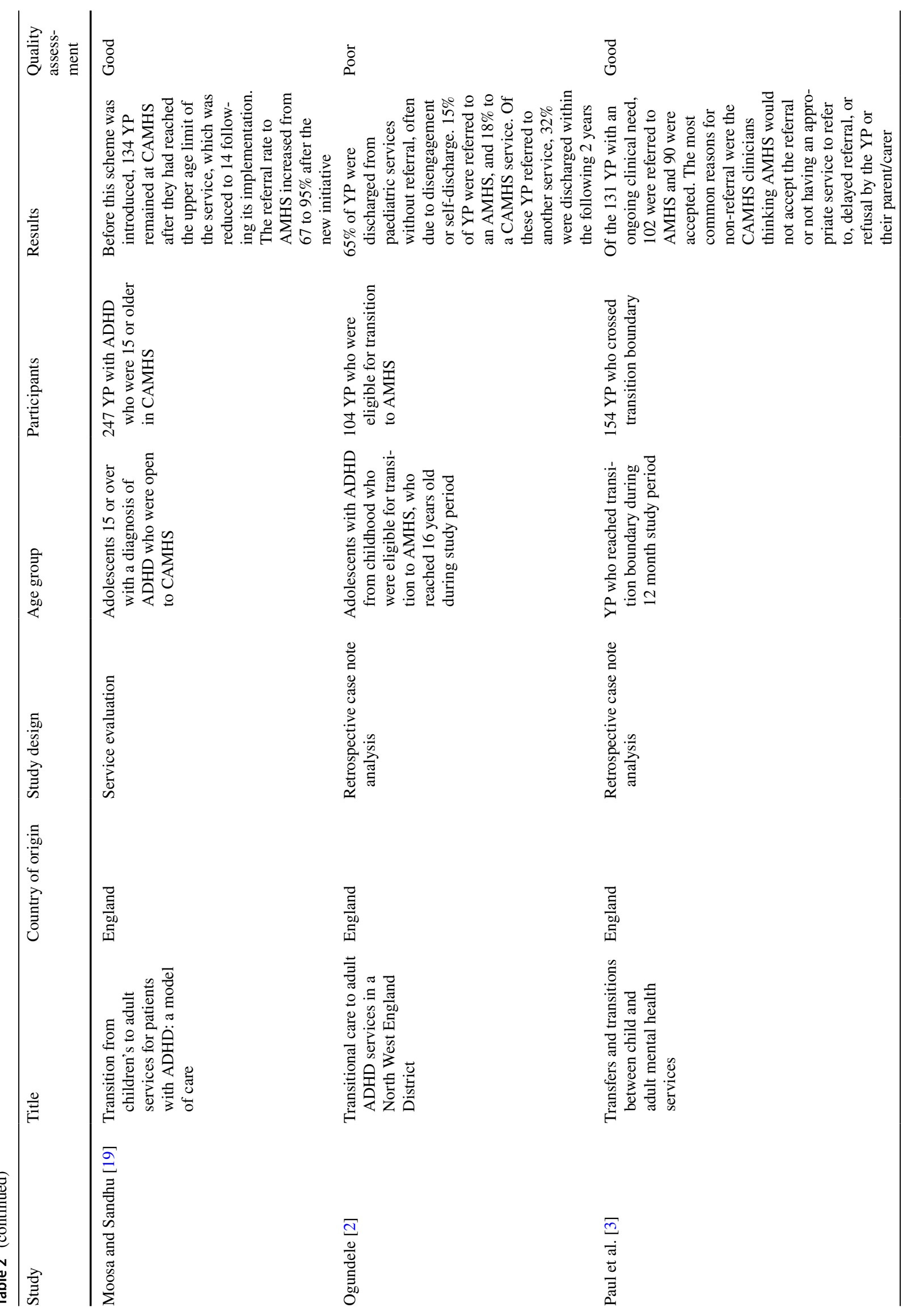




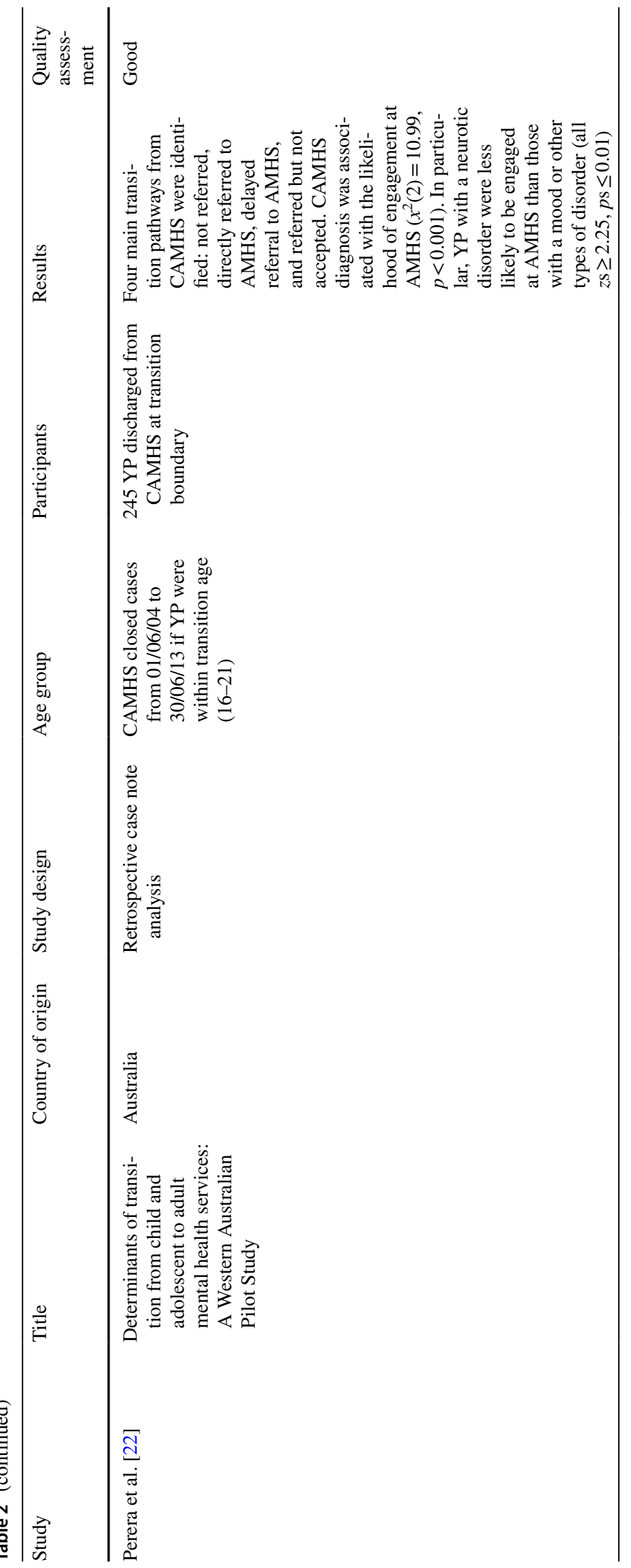




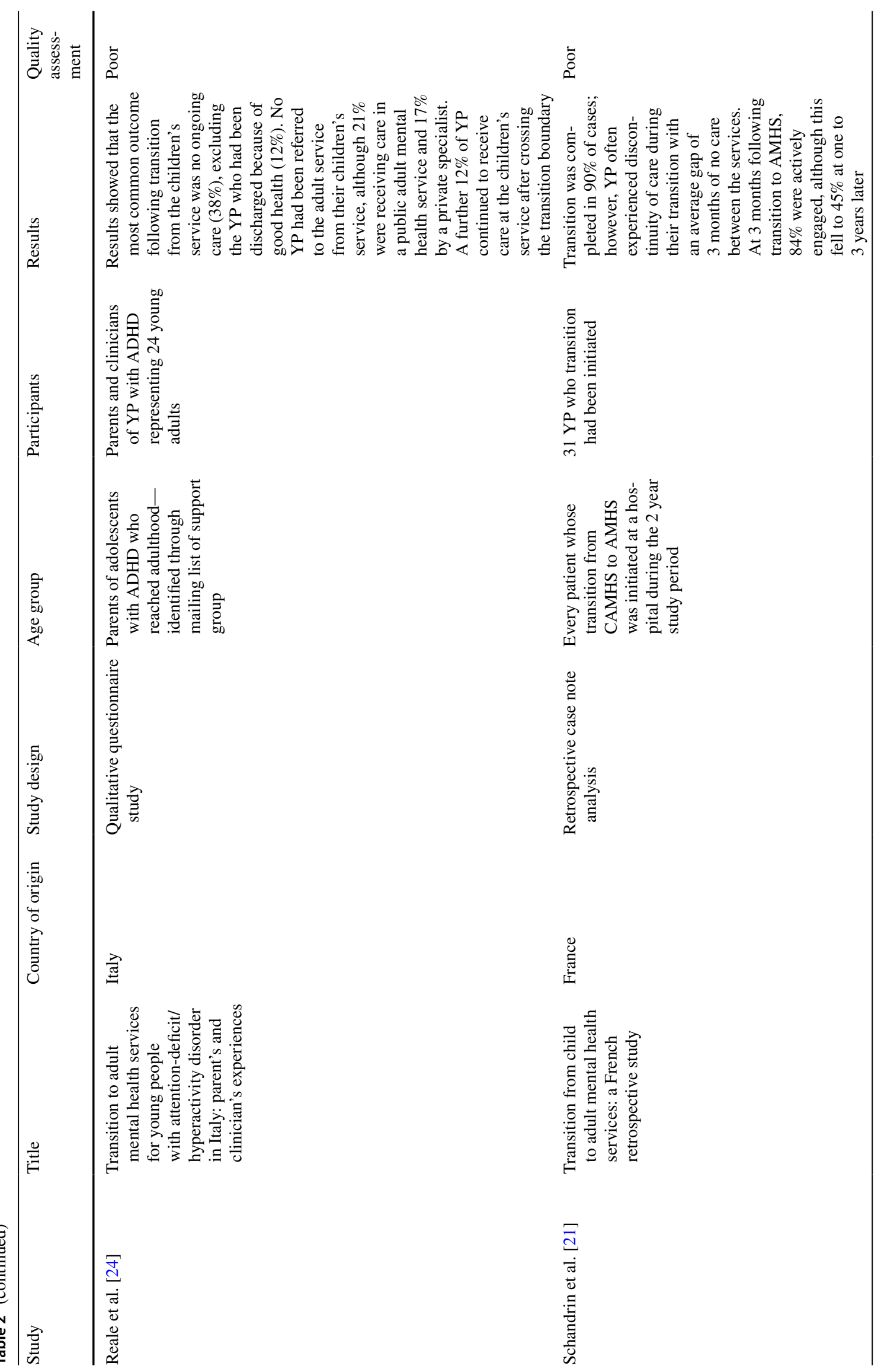




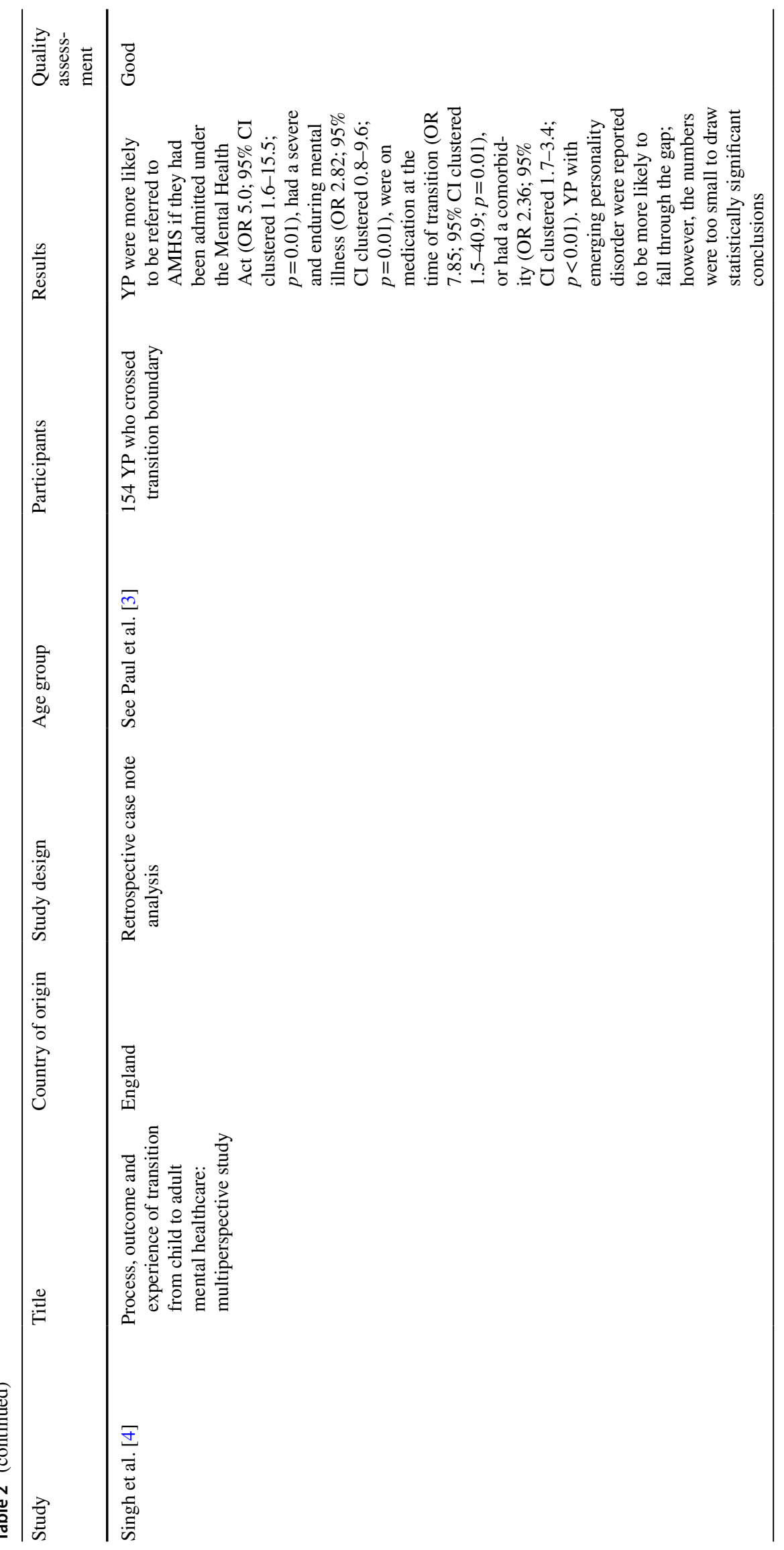




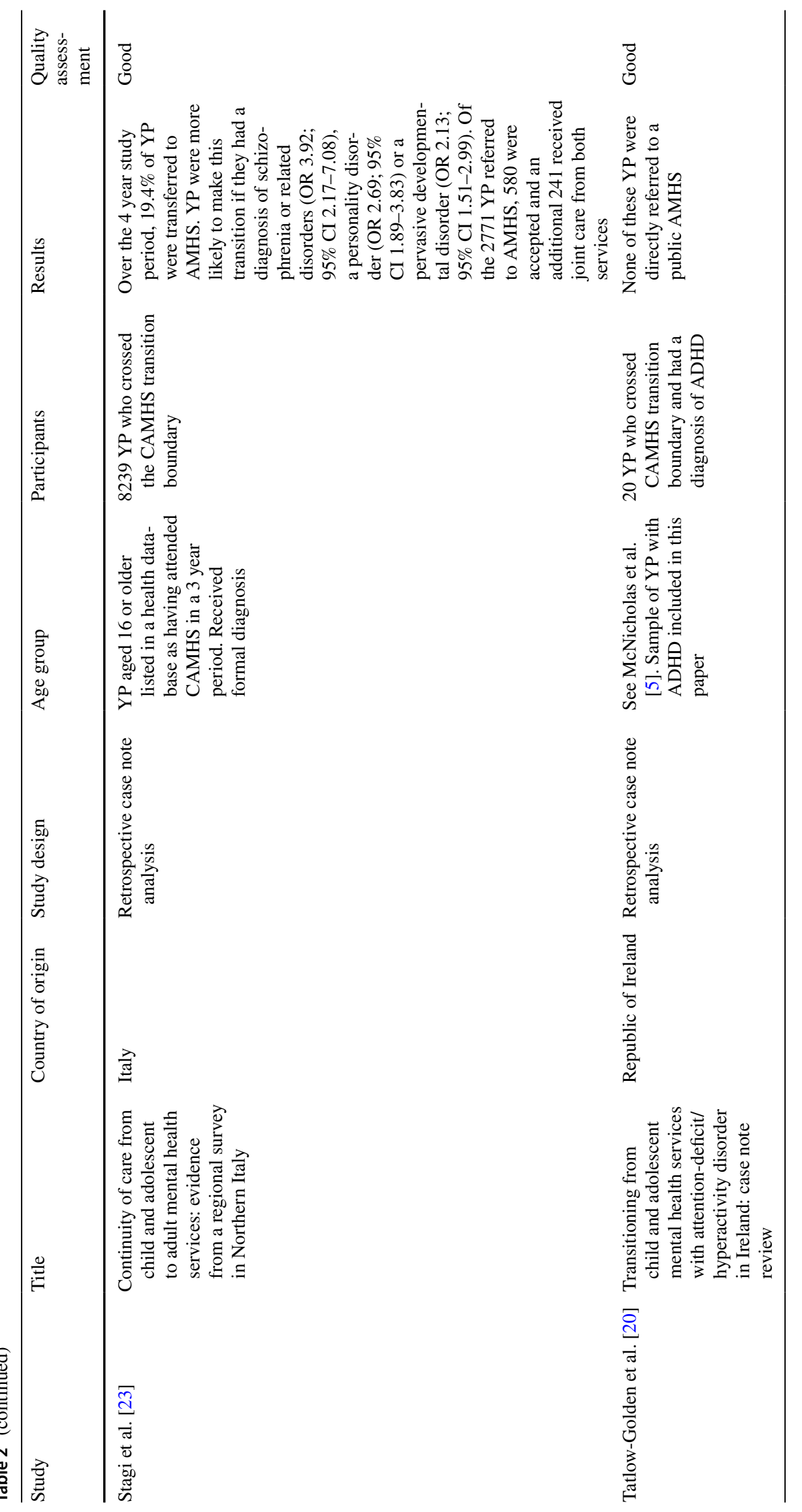




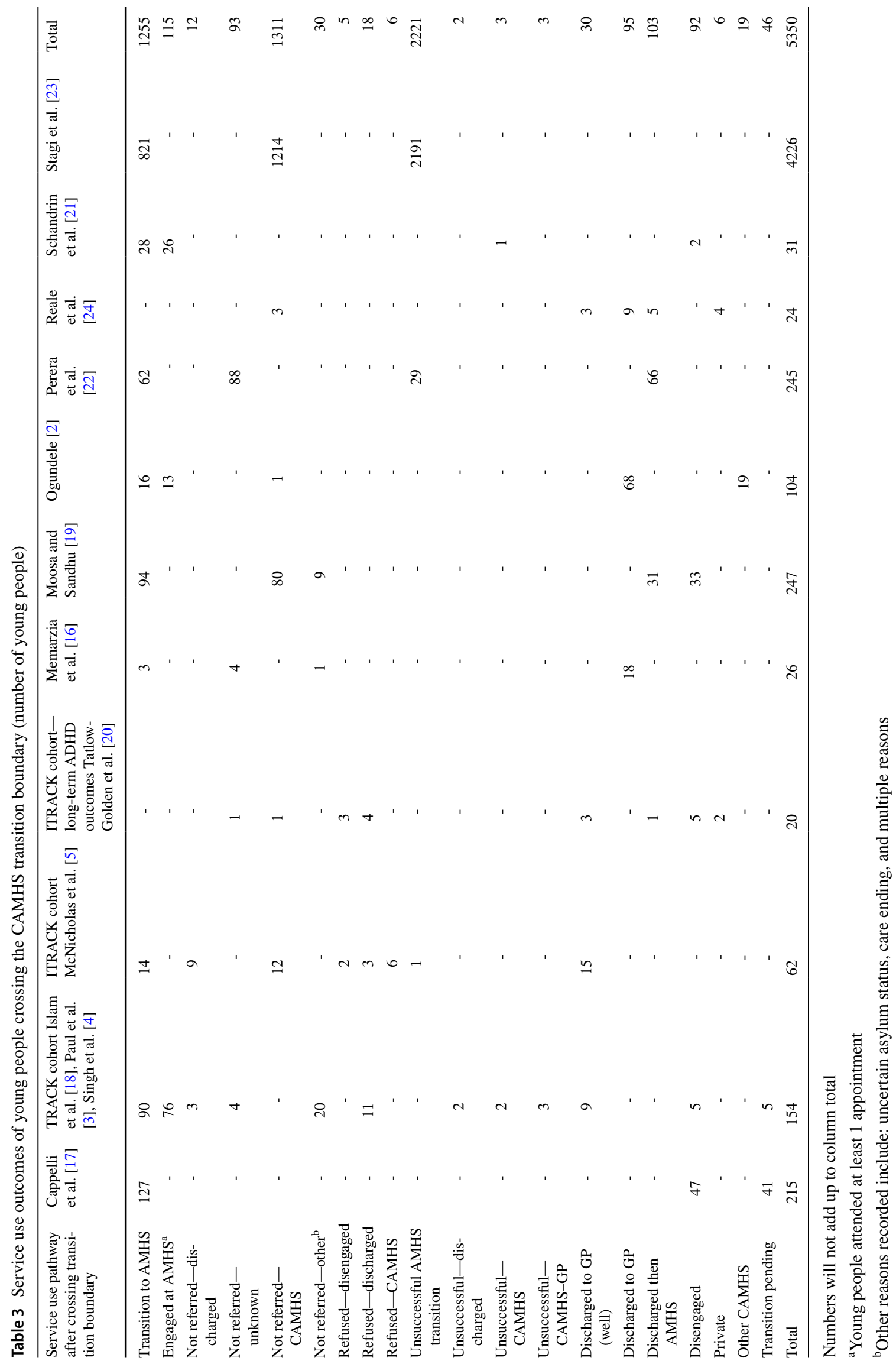


Two studies $[3,5]$ reported young people not being referred to AMHS, because CAMHS clinicians did not think that young people would meet the inclusion criteria or that AMHS did not have the necessary expertise. Five studies recorded unsuccessful referrals to AMHS [5, 18, 21-23], with percentages of referrals rejected ranging from 3 to $73 \%$. Full details of young people's service use outcomes following reaching the upper age limit of their CAMHS service is shown in Table 3.

\section{Optimal transition}

Three studies evaluated how many young people experienced optimal transition, two $[4,21]$ according to the four principles of 'optimal transition' identified by Paul et al. [3]. In most cases, optimal transition was not achieved, with percentages of young people having optimal transition recorded at $6 \%[16], 13 \%$ [21], and 4\% [4].

\section{Waiting times}

Three studies explored the average waiting times which young people experienced during their transition to AMHS $[5,17,21]$. All found that young people experienced long delays, ranging from 55 to 110 days.

\section{AMHS engagement}

Three studies looked at engagement at AMHS following transition, the TRACK study (as reported by [3, 4]), Ogundele [2], and Schandrin et al. [21]. Of the 134 young people in these studies who transitioned to AMHS, $115(86 \%)$ had at least one appointment. Rates of engagement fell further after this first appointment, with $16 \%$ being discharged after one AMHS appointment in the TRACK study [4] and 55\% being discharged in the 1-3 years following transition in the study by Schandrin et al. [21].

\section{Outcomes of young people with ADHD}

Four studies focused on young people with ADHD. One was a service evaluation following improvements to their transition process [19], and this showed a much higher rate of transition to AMHS (38\%) than the other three studies carried out in the standard care (11\%). In two of the studies involving young people with ADHD, none of the cohort was transitioned to an AMHS at the CAMHS age boundary [20, 24]. Of the young people who were discharged to their GP following cessation of care in CAMHS, one-third were then referred to an AMHS, implying that they were discharged despite having an ongoing clinical need for treatment.

\section{Discussion}

The aim of this review was to synthesise the existing research on mental health and service use outcomes of young people after leaving CAMHS. Thirteen studies were included, all of which reported service use destinations of young people after leaving CAMHS. Only one study included mental health outcomes after transition; however, as these data were reported for the whole cohort which included young people leaving care, this review focuses on service use outcomes after leaving CAMHS.

The included studies show the wide range of service use destinations of young people who reach the upper age limit of CAMHS, with only around a quarter of young people continuing care in AMHS. Alternative destinations included: other CAMHS services, community-based services, private care, or transfer of care to a GP. There are a variety of different pathways taken by young people, and multiple changes of service are common during this transition period. A quarter of young people stayed in CAMHS despite reaching the upper age limit of that service, either due to non-referral or their referral to AMHS not being accepted. This high variability in transition outcomes reflects the different ways which CAMHS services are funded and organised in different countries, as well as the availability of appropriate AMHS [1, 26]. In addition to variation between countries, there was also significant variation in outcomes between participants studied at a national level, in the United Kingdom. These results indicate that young people receive differing quality of care depending on where they live, with different service models and transition boundaries.

There was also evidence to show that some young people experienced high disruption during the transition period: some were not referred onwards despite still requiring treatment when they crossed the CAMHS age boundary, whilst very few of those who did transition received optimal transitional care. This suggests that young people were poorly prepared for transition and experienced poor continuity of care, something echoed in several research studies exploring young people's experiences of transition (e.g., [7, 25]). Having a poor transition experience could result in poor engagement with the adult service [11], which is supported by the findings in this review as studies showed the high levels of disengagement. Young people may also find it difficult to engage with AMHS due to the significant difference in focus and culture between the two services, something which has been identified as a potential barrier to young people's engagement in continued mental health care [10]. The results of this review suggest that services are not following the current guidance for the best practice, which states that transition planning should be started early and in conjunction with the young person, whilst taking into account their 
need for ongoing support and at what point transition would be most appropriate [13]. Moving forward, services should aim to align clinical practice with the current mental health policy to provide the best possible care for young people as they reach the upper age limit of CAMHS.

Four of the included studies focused on young people with ADHD, as young people with this diagnosis are among the groups least likely to transition to AMHS [4]. In two of these studies, none of the young people were transitioned directly to AMHS, although a minority were referred to adult services by their GP or received private care after leaving CAMHS [20, 24]. This could reflect a lack of appropriate service provision in some areas, leaving CAMHS with no choice but to discharge the young person to their GP [27]. In contrast, the service improvement study by Moosa and Sandhu [19] reported much higher rates of transition, suggesting that AMHS will accept the referrals of young people with ADHD, providing that the transition is managed effectively.

As several young people were not transitioned directly to AMHS, but, instead, first discharged to a GP, it can be argued that they did not receive sufficient continuity of care during their transition between services. Studies did not explore why a direct transfer of care was not made. A further clinical implication of this review is the finding that around a quarter of young people studied remained at CAMHS, even after reaching the upper age limit for that service. In this case, CAMHS should receive the appropriate funding and resources to provide this ongoing care, without restricting their ability to accept new referrals. One way in which mental health services have responded to the need for streamlined care has been to introduce new 14-25 services, removing the traditional transition boundary at around 16-18 years of age [28]. The initial findings have indicated that this new service model can help to reduce the number of young people experiencing an abrupt end to their care when they reach 18 [29]. However, in order for these services to operate effectively, appropriate funding and resources are needed to ensure that other service users do not suffer as a result.

\section{Implications for future research}

This review has also highlighted gaps in the existing research regarding service use outcomes of young people who reach the upper age limit of CAMHS, in particular longitudinal research which includes longer term outcomes in the months or years after transition. In recent years, new transition guidelines have been released; however, we are unable to fully assess what impact these guidelines have had on clinical practice due to the lack of research in this area. More longitudinal research is required to fully understand how these guidelines have been incorporated into practice and what impact they have had on the transition experiences of young people. The mental health outcomes of young people following transition are also currently unknown, something which should be made a priority in future research.

\section{Strengths and limitations}

To our knowledge, this is the first review which has systematically synthesised evidence for the service use destinations of young people after they have reached the upper age limit of CAMHS. This review has systematically collated and critically evaluated transition research from six different countries, giving a picture of transition outcomes across high-income countries. A particular strength of the methodology employed was the use of wide search criteria to minimise chances of missing relevant research. Searches also included grey literature and had no language restrictions. However, not all of the studies included were of a high methodological quality, and therefore, there are some limitations which should be considered during the interpretation of these results.

First, poor record keeping by the mental health services in some of the studies meant that the service use outcomes of some cases were unknown. Poor record keeping in some services also led to differences in the selection method of cases; some used record linkage, whilst others used clinicians to retrospectively identify eligible cases as records were not available. It is possible that cases with a particularly good or bad transition were more likely to be remembered which could lead to bias in the sample. A further limitation is that some studies did not report long-term outcomes; for example, they did not show what happened to young people whose transition was recorded as 'pending', those who stayed in CAMHS, or those whose referral to AMHS was unsuccessful.

Details about a young person's mental health and illness severity were also missing from some studies. For example, not all studies evaluated ongoing clinical need at the transition boundary; in some cases, this was not mentioned, whilst, in others, having a diagnosis of mental illness was enough to imply an ongoing need. Therefore, we cannot draw firm conclusions regarding the true numbers of young people who were not transitioned to AMHS despite still being unwell and needing further care. Similarly, not all studies distinguished between young people who were discharged to their GP, because they were well and so no longer needed treatment, those discharged to GP for continued medical review, and those who were discharged to their GP, because there was no appropriate service for them to transition to.

Finally, heterogeneity between the analyses in the different studies meant that quantitative synthesis of results using a meta-analysis was not appropriate. 


\section{Conclusions}

Systematic review of the literature revealed that only a quarter of young people continued to access care at AMHS after reaching the upper age limit of CAMHS. The remainders have varied service use outcomes, characterised by multiple transitions during this period. Future research should record the long-term outcomes of CAMHS leavers, both in terms of whether they continue to receive care and their mental health and functioning outcomes after transition.

Acknowledgements I would like to thank Frédérick Russet, Ariel Wang, and Muna Dubad for their help with the translation of some of the papers into English.

\section{Compliance with ethical standards}

Conflict of interest On behalf of all authors, the corresponding author states that there is no conflict of interest.

Open Access This article is distributed under the terms of the Creative Commons Attribution 4.0 International License (http://creativeco mmons.org/licenses/by/4.0/), which permits unrestricted use, distribution, and reproduction in any medium, provided you give appropriate credit to the original author(s) and the source, provide a link to the Creative Commons license, and indicate if changes were made.

\section{Appendix}

A breakdown of quality assessment scores for each paper Table 4 . The number of stars etc.

Table 4 The number of stars scored in each domain of the Newcastle-Ottawa scale for each included paper

\begin{tabular}{llll}
\hline Study & Selection $(* / 4)$ & $\begin{array}{l}\text { Compa- } \\
\text { rability } \\
(* / 2)\end{array}$ & Outcome $(* / 3)$ \\
\hline Cappelli et al. [17] & $3^{*}$ & $2^{*}$ & $2^{*}$ \\
Islam et al. [18] & $3^{*}$ & $2^{*}$ & $3^{*}$ \\
McNicholas et al. [5] & $3^{*}$ & $2^{*}$ & $3^{*}$ \\
Memarzia et al. [16] & $4^{*}$ & $1^{*}$ & $2^{*}$ \\
Moosa and Sandhu [19] & $3^{*}$ & $2^{*}$ & $3^{*}$ \\
Ogundele [2] & $3^{*}$ & $0^{*}$ & $3^{*}$ \\
Paul et al. [3] & $3^{*}$ & $2^{*}$ & $3^{*}$ \\
Perera et al. [22] & $4^{*}$ & $1^{*}$ & $3^{*}$ \\
Reale et al. [24] & $2^{*}$ & $0^{*}$ & $1^{*}$ \\
Schandrin et al. [21] & $4^{*}$ & $0^{*}$ & $2^{*}$ \\
Singh et al. [4] & $3^{*}$ & $2^{*}$ & $3^{*}$ \\
Stagi et al. [23] & $3^{*}$ & $1^{*}$ & $2^{*}$ \\
Tatlow-Golden et al. & $3^{*}$ & $2^{*}$ & $3^{*}$ \\
[20] & & & \\
\hline
\end{tabular}

\section{References}

1. Signorini G, Singh SP, Marsanic VB, Dieleman G, DodigĆurković K, Franic T et al (2018) The interface between child/ adolescent and adult mental health services: results from a European 28-country survey. Eur Child Adolesc Psychiatry 27:501-511

2. Ogundele MO (2013) Transitional care to adult ADHD services in a North West England district. Clin Gov Int J 18:210-219

3. Paul M, Ford T, Kramer T, Islam Z, Harley K, Singh SP (2013) Transfers and transitions between child and adult mental health services. Br J Psychiatry 202:s36-s40

4. Singh SP, Paul M, Ford T, Kramer T, Weaver T, McLaren S et al (2010) Process, outcome and experience of transition from child to adult mental healthcare: multiperspective study. Br J Psychiatry 197:305-312

5. McNicholas F, Adamson M, McNamara N, Gavin B, Paul M, Ford T et al (2015) Who is in the transition gap? Transition from CAMHS to AMHS in the Republic of Ireland. Ir J Psychol Med 32:61-69

6. Belling R, McLaren S, Paul M, Ford T, Kramer T, Weaver T et al (2014) The effect of organisational resources and eligibility issues on transition from child and adolescent to adult mental health services. J Health Serv Res Policy 19:169-176

7. Dunn V (2017) Young people, mental health practitioners and researchers co-produce a Transition Preparation Programme to improve outcomes and experience for young people leaving Child and Adolescent Mental Health Services (CAMHS). BMC Health Serv Res 17:293

8. Vyas N, Birchwood M, Singh SP (2015) Youth services: meeting the mental health needs of adolescents. Ir J Psychol Med 32:13-19

9. Singh SP (2009) Transition of care from child to adult mental health services: the great divide. Curr Opin Psychiatry 22:386-390

10. Birchwood M, Singh SP (2013) Mental health services for young people: matching the service to the need. Br J Psychiatry Suppl 54:s1-s2

11. Mulvale GM, Nguyen TD, Miatello AM, Embrett MG, Wakefield PA, Randall GE (2015) Lost in transition or translation? Care philosophies and transitions between child and youth and adult mental health services: a systematic review. J Ment Health. https ://doi.org/10.3109/09638237.2015.1124389

12. Healthcare Safety Investigation Branch (2018) Investigation into the transition from child and adolescent mental health services to adult mental health services. https://www.hsib.org.uk/investigat ions-cases/transition-from-child-and-adolescent-mental-healt hservices-to-adult-mental-health-services/final-report/. Accessed 20 July 2018

13. NICE (2016) Transition from children's to adults' services for young people using health or social care services. National Institute for Health and Care Excellence, London

14. Wells G, Shea B, O'Connell D, Petersen J, Welch V, Losos M et al (2011) The Newcastle-Ottawa Scale (NOS) for assessing the quality of nonrandomized studies in meta-analyses. Department of Epidemiology and Community Medicine, University of Ottawa, Canada. University of Ottawa, Canada. http://www.ohri.ca/progr ams/clinical_epidemiology/oxford.asp. Accessed 20 Nov 2017

15. Popay J, Roberts H, Sowden A, Petticrew M, Arai L, Rodgers M et al (2006) Guidance on the conduct of narrative synthesis in systematic reviews. A product from the ESRC methods programme, vol 10(2.1). Lancaster University, Lancaster, pp 1018-4643

16. Memarzia J, St Clair MC, Owens M, Goodyer IM, Dunn VJ (2015) Adolescents leaving mental health or social care services: predictors of mental health and psychosocial outcomes one year later. BMC Health Serv Res 15:185 
17. Cappelli M, Davidson S, Racek J, Leon S, Vloet M, Tataryn K et al (2016) Transitioning youth into adult mental health and addiction services: an outcomes evaluation of the youth transition project. J Behav Health Serv Res 43:597-610

18. Islam Z, Ford T, Kramer T, Paul M, Parsons H, Harley K et al (2016) Mind how you cross the gap! Outcomes for young people who failed to make the transition from child to adult services: the TRACK study. BJPsych Bull 40:142-148

19. Moosa F, Sandhu TJ (2015) Transition from children's to adult services for patients with ADHD: a model of care. Adolesc Psychiatry 5:22-30

20. Tatlow-Golden M, Gavin B, McNamara N, Singh S, Ford T, Paul $M$ et al (2017) Transitioning from child and adolescent mental health services with attention-deficit hyperactivity disorder in Ireland: case note review. Early Interv Psychiatry 12:505-512

21. Schandrin A, Capdevielle D, Boulenger J-P, Batlaj-Lovichi M, Russet F, Purper-Ouakil D et al (2016) Transition from child to adult mental health services: a French retrospective survey. J Ment Health Train Educ Pract 11:286-293

22. Perera RH, Rogers SL, Edwards S, Hudman P, Malone C (2017) Determinants of transition from child and adolescent to adult mental health services: a western Australian pilot study. Aust Psychol 52:184-190

23. Stagi P, Galeotti S, Mimmi S, Starace F, Castagnini AC (2015) Continuity of care from child and adolescent to adult mental health services: evidence from a regional survey in Northern Italy. Eur Child Adolesc Psychiatry 24:1535-1541
24. Reale L, Frassica S, Gollner A, Bonati M (2015) Transition to adult mental health services for young people with attention deficit hyperactivity disorder in Italy: parents' and clinicians' experiences. Postgrad Med 127:671-676

25. Hovish K, Weaver T, Islam Z, Paul M, Singh SP (2012) Transition experiences of mental health service users, parents, and professionals in the United Kingdom: a qualitative study. Psychiatr Rehabil J 35:251

26. Signorini G, Singh SP, Boricevic-Marsanic V, Dieleman G, Dodig-Ćurković K, Franic T et al (2017) Architecture and functioning of child and adolescent mental health services: a 28-country survey in Europe. Lancet Psychiatry 4:715-724

27. Hall CL, Newell K, Taylor J, Sayal K, Hollis C (2015) Services for young people with attention deficit/hyperactivity disorder transitioning from child to adult mental health services: a national survey of mental health trusts in England. J Psychopharmacol 29:39-42

28. Wilson J, Clarke T, Lower R, Ugochukwu U, Maxwell S, Hodgekins J et al (2018) Creating an innovative youth mental health service in the United Kingdom: the Norfolk Youth Service. Early Interv Psychiatry 12:740-746

29. Maxwell S, Ugochukwu O, Clarke T, Gee B, Clarke E, Westgate H, Wilson J, Lenno BR, Goodyer IM (2019) The effect of a youth mental health service model on access to secondary mental healthcare for young people aged 14-25 years. BJPsych Bull 43:27-31 